\title{
A Novel Adaptive Fuzzy Variable Structure Control for a Class of Nonlinear Uncertain Systems via Backstepping
}

\author{
Feng-Yih $\mathrm{Hsu}^{1}$ and Li-Chen $\mathrm{Fu}^{1,2}$ \\ Dept. of Electrical Engineering ${ }^{1}$ \\ Dept. of Computer Science \& Information Engineering ${ }^{2}$ \\ National Taiwan University, Taipei, Taiwan, R.O.C.
}

\begin{abstract}
This paper presents a new design of adaptive fuzzy variable structure control to solve the traditional problem of model reference adaptive control (MRAC) for a class of single-input, single-output minimum-phase uncertain nonlinear systems via backstepping. Instead of taking the tedious coordinate transformation and yielding a "hard" high-gain controller, we introduce smooth B-spline-type membership functions. into the controller so as to compensate for the uncertainties much "softer", i.e., in a much smoother and locally weighted manner. To be rigorous, it is shown that the stability of the closed-loop system can be assured and the tracking error can globally approach to an arbitrary preset dead-zone range.
\end{abstract}

\section{Introduction}

Based on the differential geometrical approach, most recent research works on affine nonlinear systems involve system linearization, i.e. to adaptively cancel the unmodeled terms which can be linearly parameterizable [5]. For solving the state-feedback linearizing contrci problems, the adaptive backstepping designs have been developed, e.g., [6]-[7]. To cope with the output feedback control problems, in which the system nonlinearity depends solely on the output, a filtered transformation and a backstepping approach were employed to yield global asymptotical stability either through some linear parameterization process [1],[8] or nonlinear one [2]. It turns out that the latter nonlinear parameterization characterizes a more general class of the nonlinear systems.

On the other hand, the fuzzy variable structure control can provide the stability and smoothness at the same time of a fuzzy control if the fuzzy control is formulated in a form of variable structure control [16]-[18], or if a variable structure control is augmented with some ruleparameter setting mechanism [19]-[22]. However, a systematic approach is lacking for enhancing the smoothess of a robust fuzzy control design. In this paper, an adaptive fuzzy variable structure control with smooth membership functions and using backstepping concept is hereby systematically developed to yield improved tracking performance relative to that from the high-gain con- troller. Based on approximation theory, several kinds of basis function such as sinusoid basis, or Gaussian basis functions [23], [24], are often incorporated into an adaptive controller. In this paper, we choose the smooth $\mathrm{B}$-spline basis functions as the membership functions in the paradigm of fuzzy approximation [25]. This choice is due to the fact that nonlinear functions whose feature are mostly strongly local can be represented by some kind of wavelet transform characterized by smooth and compact support [26], [28]. Given such smooth B-splinetype membership functions, the proposed adaptive fuzzy variable structure controller with a dedicated structure can adaptively compensate for the system uncertainties, in a smooth and locally weighted manner, but not to evolve into a global "hard" high-gain controller [3].

\section{Problem Formulation}

Consider an affine nonlinear system of the form as follows:

$$
\begin{aligned}
\dot{x} & =f(x, \alpha)+g(x, \alpha) u \\
y & =h(x, \alpha)
\end{aligned}
$$

where $x \in \Re^{n}, u \in \Re, y \in \Re$, and $\alpha\left(\in \Omega_{\alpha}\right.$ : a compact set) is an unknown constant parameter vector which characterizes the smooth nonlinear function vectors $f$, $g$, and scalar $h$, satisfying $f(0, \alpha)=0, g(0, \alpha) \neq 0$ and $h(0, \alpha)=0, \forall \alpha \in \Omega_{\alpha}$.

If the nonlinear system described in (1) can satisfy some geometric coordinate-free conditions [1], then there exists a coordinate transformation $z=T(x)$ such that the nonlinear system can be transformed into the following output-feedback form:

$$
\begin{aligned}
\dot{z} & =A z+\Phi(y, \alpha)+b(\alpha) \delta(y) u \\
y & =c^{T} z
\end{aligned}
$$

where

$$
\begin{gathered}
A=\left[\begin{array}{ccccc}
0 & 1 & 0 & \ldots & 0 \\
0 & 0 & 1 & \ldots & 0 \\
\vdots & \vdots & \vdots & \ddots & \vdots \\
0 & 0 & 0 & \ldots & 1 \\
0 & 0 & 0 & \ldots & 0
\end{array}\right], \Phi(y, \alpha)=\left[\begin{array}{c}
\Phi_{1}(y, \alpha) \\
\vdots \\
\Phi_{n}(y, \alpha)
\end{array}\right], \\
b(\alpha)=\left[\begin{array}{c}
b_{1}(\alpha) \\
\vdots \\
b_{n}(\alpha)
\end{array}\right], c=\left[\begin{array}{c}
1 \\
0 \\
\vdots \\
0
\end{array}\right]
\end{gathered}
$$

The coordinate-free conditions can be summarized as follows [1]: For any $\alpha \in \Omega_{\alpha}$, 
- $\operatorname{rank}\left\{d h, d(L, h), \cdots, d\left(L_{h}^{n-1}\right)\right\}=n$

- $\left[a d_{f}^{i} r, a d_{f}^{j} r\right]=0,0 \leq i, j \leq n-1$;

- $\left[g, a d_{f}^{k} r\right]=0,0 \leq k \leq n-2$;

- $g=\sigma(\cdot) \sum_{j=1}^{n} b_{j}(\alpha)(-1)^{n-j} a d_{f}^{n-j} r$;

- the vector fields $a d_{f}^{i} r, 0 \leq i \leq n-1$, are complete, where $r$ is the vector field satisfying

$$
L_{r} L_{f}^{j} h= \begin{cases}0, & \text { if } j=0,1, \ldots, n-2 \\ 1, & \text { if } j=n-1\end{cases}
$$

Furthermore, the system (1) is with strong relative degree $\rho$, i.e.,

$$
\begin{aligned}
L_{g} L_{f}^{i} h(x, \alpha) & =0,0 \leq i \leq \rho-2, \forall x \in \Re^{n}, \forall \alpha \in \Omega_{\alpha} \\
L_{g} L_{f}^{\rho-1} h(x, \alpha) & \neq 0, \forall \alpha \in \Omega_{\alpha}
\end{aligned}
$$

Here, our control goal is to force the output $y$ to follow a desired trajectory $y_{m}$, constructed by the following reference model:

$$
\begin{aligned}
\dot{z}_{m} & =A_{c} z_{m}+b_{m} r_{m} \\
y_{m} & =c^{T} z_{m}
\end{aligned}
$$

where

$$
A_{c}=\left[\begin{array}{ccccc}
-a_{1} & 1 & 0 & \ldots & 0 \\
-a_{2} & 0 & 1 & \ldots & 0 \\
\vdots & \vdots & \vdots & \ddots & \vdots \\
-a_{n-1} & 0 & 0 & \ldots & 1 \\
-a_{n} & 0 & 0 & \ldots & 0
\end{array}\right]
$$

is a stable Hurwitz matrix, $b_{m}=\left[0, \ldots, b_{m_{\rho}}, \ldots, b_{m n}\right]^{T}$ and $r_{m} \in L_{\infty}$ is a bounded reference input, or $y_{m}=$ $W_{m}(s) r_{m}$ with $W_{m}(s)=\frac{b_{m} s^{n-\rho}+\cdots+b_{m n}}{s^{n}+a_{1} s^{n-1}+\cdots+a_{n}}$ so that $y_{m} \in$ $L_{\infty}$.

In order to make the model tracking problem more tractable, we make the following reasonable assumptions.

\section{Assumptions:}

(A1) $b=\left[b_{1}, \ldots, b_{n}\right]^{T}$ is a vector of Hurwitz coefficients of degree $\rho$, i.e., the associated polynomial

$$
b_{1} s^{n-1}+b_{2} s^{n-2}+\cdots+b_{n}
$$

is of degree $n-\rho$ (i.e., $b_{1} \neq 0$ if $\rho=1$ or $b_{1}=\cdots=b_{\rho-1}=$ $0, b_{\rho} \neq 0$, if $\rho>1$ ) and is Hurwitz;

(A2) The sign of $b_{\rho}(\alpha)$ is known and constant for any $\alpha \in \Omega_{\alpha}$, $1 \leq \rho \leq n$

(A3) $\Phi(y, \alpha)$ can be expressed as a Taylor's series expansion in $y$ for any $\alpha \in \Omega_{\alpha}$, i.e., $\Phi(y, \alpha)=\Phi\left(y_{0}, \alpha\right)+$ $\sum_{n=1}^{\infty}\left[\left.\frac{1}{n !} \frac{\partial^{(n)} \Phi}{\partial y^{n}}\right|_{y=y_{0}}\left(y-y_{0}\right)^{n}\right]$

(A4) $\left\|\Phi(y, \alpha)-\Phi\left(y_{0}, \alpha\right)\right\| \leq \iota_{\Phi}\left(y-y_{0}, y_{0}, \alpha\right)\left|y-y_{0}\right|$, for some $l_{\Phi}\left(y-y_{0}, y_{0}, \alpha\right) \geq 0$.

Given such tracking problem, we proceed with rearranging the form (2) by the following:

$$
\begin{aligned}
\dot{z} & =A z-a y+a y+\Phi(y, \alpha)+b(\alpha) \delta u \\
& =A_{c} z+a y+\Phi(y, \alpha)+b(\alpha) \delta u \\
y & =c^{T} z
\end{aligned}
$$

where $a=\left[a_{1}, a_{2}, \ldots, a_{n}\right]^{T}$ and let $W(s)$ be defined as

$$
W(s)=c^{T}\left(s I-A_{c}\right)^{-1} b=\frac{b_{\rho} s^{n-\rho}+\cdots+b_{n}}{s^{n}+a_{1} s^{n-1}+\cdots+a_{n}}
$$

which apparently is a stable transfer function. Define the error vector $e=z_{m}-z$, then the error model can be derived as follows:

$$
\begin{aligned}
\dot{e} & =A_{c} e-a y-\Phi+b_{m} r_{m}-b \delta(y) u \\
e_{o} & =y_{m}-y=c^{T} e
\end{aligned}
$$

\section{Robust Output-Feedback Variable Structure Control via Backstepping}

Consider the case of relative degree $\rho>1$ ( the case of $\rho=1$ can be intuitively derived ) and a stable filter:

$$
W_{f}^{-1}(s)=\frac{1}{\left(s+\lambda_{1}\right)\left(s+\lambda_{2}\right) \cdots\left(s+\lambda_{\rho-1}\right)}, \lambda_{i}>0
$$

characterizing the input-output relationship $\eta_{1}=W_{f}^{-1}(s) \delta(y) u$, which is realized into the state space form as follows:

$$
\dot{\eta}=A_{f} \eta+b_{f} \delta(y) u, \eta \in \Re^{\rho-1}
$$

where

$$
A_{f}=\left[\begin{array}{ccccc}
-\lambda_{1} & 1 & 0 & \ldots & 0 \\
0 & -\lambda_{2} & 1 & \ldots & 0 \\
\vdots & \vdots & \vdots & \ddots & \vdots \\
0 & 0 & 0 & \ldots & 1 \\
0 & 0 & 0 & \ldots & -\lambda_{\rho-1}
\end{array}\right], b_{f}=\left[\begin{array}{c}
0 \\
\vdots \\
1
\end{array}\right]
$$

$\eta=\left[\eta_{1}, \ldots, \eta_{\rho-1}\right]$, and $\eta(0)=\eta_{0}$. Then, augment the error model (6) with this filter (8) as follows:

$$
\begin{aligned}
{\left[\begin{array}{l}
\dot{e} \\
\eta
\end{array}\right] } & =\left[\begin{array}{cc}
A_{c} & 0 \\
0 & A_{f}
\end{array}\right]\left[\begin{array}{l}
e \\
\eta
\end{array}\right]+\left[\begin{array}{c}
-b(\alpha) \\
b_{f}
\end{array}\right] \delta(y) u+\left[\begin{array}{c}
-a y-\Phi(y, \alpha)+b_{m} r_{m} \\
0
\end{array}\right] \\
e_{0} & =c^{T_{e}}
\end{aligned}
$$

which is equivalent to the following:

$$
\begin{aligned}
\dot{\zeta} & =A_{c} \zeta-a y-\Phi(y, \alpha)+b_{m} r_{m}-d(\alpha) \eta_{1} \\
e_{o} & =c^{T} \zeta
\end{aligned}
$$

from the I/O point of view, where $d=\left[d_{1}, \cdots, d_{n}\right]$ is a vector of Hurwitz coefficients of degree one, derived from the following transfer function

$$
\begin{aligned}
W(s) W_{f}(s) & =\frac{b_{\rho} s^{n-p}+\cdots+b_{n}}{s^{n}+a_{1} s^{n-1}+\cdots+a_{n}}\left(s+\lambda_{1}\right)\left(s+\lambda_{2}\right) \cdots\left(s+\lambda_{\rho-1}\right) \\
& =\frac{d_{1} s^{n-1}+\cdots+d_{n}}{s^{n}+a_{1} s^{n-1}+\cdots+a_{n}}
\end{aligned}
$$

Apparently, $d_{1}=b_{\rho}$. After applying the transformation developed by [2], we define a new vector $\xi=\left[\xi_{1}, \cdots, \xi_{n-1}\right]^{T}$ as follows:

$$
\begin{aligned}
\xi_{1} & =\zeta_{2}-\frac{d_{2}(\alpha)}{d_{1}(\alpha)} e_{o} \\
& \vdots \\
\xi_{n-1} & =\zeta_{n}-\frac{d_{n}(\alpha)}{d_{1}(\alpha)} e_{o},
\end{aligned}
$$

whereby we can obtain a different dynamic model as shown below:

$$
\xi=\left[\begin{array}{ccccc}
-\frac{d_{2}}{d_{1}} & 1 & 0 & \cdots & 0 \\
-\frac{d_{3}}{d_{1}} & 0 & 1 & \cdots & 0 \\
\vdots & \vdots & \vdots & \ddots & \vdots \\
-\frac{d_{n-1}}{d_{1}} & 0 & 0 & \cdots & 1 \\
-\frac{d_{n}}{d_{1}} & 0 & 0 & \cdots & 0
\end{array}\right] \xi+\left[\begin{array}{c}
\frac{d_{3}}{d_{1}}-\frac{d_{2}^{2}}{d_{1}^{2}} \\
\frac{d_{4}}{d_{1}}-\frac{d_{3} d_{2}}{d_{1}^{2}} \\
\vdots \\
\frac{d_{n-1} d_{2}}{d_{1}^{2}} \\
-\frac{d_{n} d_{2}}{d_{1}^{2}}
\end{array}\right] e_{0}+
$$

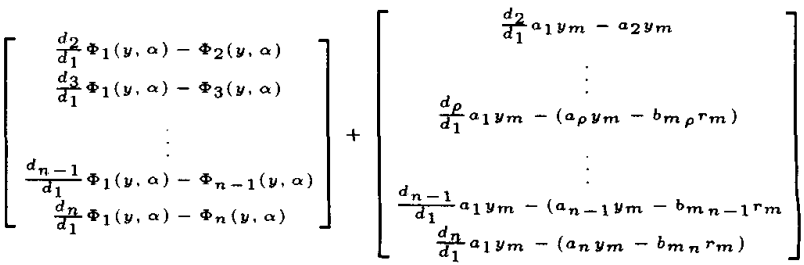


$=\Gamma \xi+\beta e_{o}+\Psi(y, \alpha)+\Psi_{r}\left(y_{m}, r_{m}\right)$

where $\Gamma$ is apparently a Hurwitz matrix. From assumption (A4), it can be easily verified that

$$
\left\|\Psi(y, \alpha)-\Psi\left(y_{m}, \alpha\right)\right\| \leq l_{\Phi}\left(e_{o}, y_{m}, \alpha\right)\left|e_{o}\right|
$$

for some $l_{\Phi}\left(e_{0}, y_{m}, \alpha\right) \geq 0$.

On the other hand, from the transformation (12) and the equation (10), the output error model can be expressed as follows:

$$
\dot{e}_{o}=\xi_{1}+\frac{d_{2}(\alpha)}{d_{1}(\alpha)} e_{o}-\phi\left(e_{o}, y_{m}, \alpha\right)-a_{1} y_{m}-d_{1} \eta_{1},
$$

where

$$
\phi\left(e_{o}, y_{m}, \alpha\right)=\Phi_{1}\left(y_{m}-e_{o}, \alpha\right)=\Phi_{1}(y, \alpha)
$$

It is well known that applying the controller design based on the backstepping procedure can solve the control problem with the error model (15) [2]. The concepts of the backstepping control are to first design the designated controller of the first desired filter state, $\eta_{1}^{*}$, which can guarantee that the output tracking error $e_{o}$ given in the equation (15) can approach zero, and then to design the designated controller of the second desired filter state, $\eta_{2}^{*}$, which can realize $\eta_{1}$ as $\eta_{1}^{*}$ subject to the equation:

$$
\dot{\eta}_{1}=-\lambda_{1} \eta_{1}+\eta_{2}
$$

Similarly, the controller design back steps to the designated control input controller $\delta(y) u=\eta_{\rho}^{*}$ so that $\eta_{\rho-1}$ can approach $\eta_{\rho-1}^{*}$.

Now, we design a variable structure controller $\eta_{1}^{*}$ as follows:

$$
\eta_{1}^{*}=\operatorname{sgn}\left(d_{1}\right) k_{1_{1}} e_{o}+\operatorname{sgn}\left(d_{1}\right) k_{12}(t) \operatorname{sgn}\left(e_{o}\right),
$$

with smooth functions $k_{11}(t)$ and $k_{12}(t)$ satisfying the following expressions:

$$
\begin{aligned}
& k_{11}(t) \geq \frac{1}{\left|d_{1}\right|}\left(\left|\frac{d_{2}}{d_{1}}\right|+\frac{\left(\frac{1}{2}+\|P\|\left(|| \beta||+l_{\Phi}\right)\right)^{2}}{q_{0}}+\rho \epsilon_{1}\right) \\
& k_{12}(t) \geq \frac{1}{\left|d_{1}\right|}\left(|| \widehat{\xi}||+|\phi|+\left|a_{1}\right|\left|y_{m}\right|\right),
\end{aligned}
$$

where $q_{0}, \epsilon_{1}>0$ are positive constants, $P$ is a positive definite matrix to be defined later, and $\|\widehat{\xi}\|$ is constructed from the following dynamic equation:

$$
\dot{\widehat{\xi}}=\Gamma \widehat{\xi}+\Psi\left(y_{m}, \alpha\right)+\Psi_{r}\left(y_{m}, r_{m}\right),
$$

with initial conditions $\widehat{\xi}(0)=\widehat{\xi}_{0}$. Apparently, the system (20) is BIBO stable, since $\Psi\left(y_{m}, \alpha\right)$ and $\Psi_{r}\left(y_{m}, r_{m}\right)$ are bounded for the bounded $y_{m}, \alpha$, and $r_{m}$, resulting in $\widehat{\xi} \in L_{\infty}^{n-1}$. This control law will be shown effective to the tracking control problem via the following proposition.

Proposition 3.1 If $\eta_{1}=\eta_{1}^{*}$ as given in the equation (18), then the output tracking error of the system (6) will be driven to zero globally and exponentially.

To realize the control law (18), apparently, the switching function $\operatorname{sgn}\left(e_{0}\right)$ will render $\eta_{1}^{*}$ to be discontinuous at $e_{o}=0$. This fact often causes $\eta_{1}^{*}$ to be unrealizable when it comes to design the subsequent designated controller $\eta_{2}^{*}$. To resolve this problem, it is straightforward to modify the previous controller by embedding a smooth compensator for a specified dead-zone range, such as a saturation-type compensator or a hyper tangent-type compensator. Then, this controller can be expressed as follows:

$\eta_{1}^{\dagger}=\operatorname{sgn}\left(d_{1}\right) k_{1}=\left\{\begin{array}{l}\left.\operatorname{sgn}\left(d_{1}\right) ! k_{1}(t) e_{o}+k_{12}(t) \operatorname{sgn}\left(e_{o}\right)\right] . \text { if } e_{0} \notin\left[-\Delta_{e}, \Delta_{e}\right] \\ k_{s}\left(e_{0}, \alpha_{1}, \Delta_{e}\right) \text { otherwise: }\end{array}\right.$

(21)

where $k_{s_{1}}(\cdot)$ is a smooth function to make $\eta_{1}^{\dagger}$ smooth, and $\left[-\Delta_{e}, \Delta_{e}\right]$ is a designated dead-zone range which can be arbitrarily set. Then, the following proposition is valid.

Proposition 3.2 If the control law $\eta_{1}=\eta_{1}^{\dagger}$ is given as in equation (21), then the tracking error of the system (6) will be driven to the dead zone range $\left[-\Delta_{e}, \Delta_{\epsilon}\right]$ globally and exponentially.
However, in fact $\eta_{1}$ is driven by $\eta_{2}$ according to the first order equation (17), and hence we define the difference between the desired smooth filter state $\eta_{1}^{\dagger}$ and the real filter state $\eta_{1}$ as $\widetilde{\eta_{1}}=\eta_{1}^{\dagger}-\eta_{1}$, so that its time derivative can be derived from (17) as follows:

$$
\dot{\widetilde{\eta_{1}}}=\dot{\eta}_{1}^{\dagger}-\dot{\eta}_{1}=\frac{\partial \eta_{1}^{\dagger}}{\partial e_{o}} \dot{e}_{o}-\lambda_{1} \tilde{\eta_{1}}+\lambda_{1} \eta_{1}^{\dagger}-\eta_{2}
$$

As a result, the goal of the control law $\eta_{2}$ is apparently to force $\eta_{1}$ to achieve $\eta_{1}^{\dagger}$ and, hence, we derive the variable structure control $\eta_{2}^{*}$ as follows:

$$
\eta_{2}^{*}=\lambda_{1} \eta_{1}^{\dagger}+k_{21}(t) \tilde{\eta_{1}}+k_{22}(t) \operatorname{sgn}\left(\tilde{\eta_{1}}\right)
$$

where smooth functions $k_{21}(\cdot)$ and $k_{22}(\cdot)$ satisfy the following conditions:

$$
\begin{aligned}
& k_{2_{1}}(t) \geq\left|d_{1}\right|\left|\frac{\partial \eta_{1}^{\dagger}}{\partial e_{0}}\right|+\frac{\left(d_{1}+\frac{d}{d} \frac{\partial \eta_{1}^{\dagger}}{\partial e_{0}}\right)^{2}}{4 \epsilon_{1}}+\frac{1}{4 \epsilon_{2}}\left(\frac{\partial \eta_{1}^{\dagger}}{\partial e_{0}}\right)^{2} \\
& k_{22}(t) \geq\left|\frac{\partial \eta_{1}^{\dagger}}{\partial e_{0}}\right|\left(|| \widehat{\xi} \|+|\phi|+\left|a_{1}\right|\left|y_{m}\right|+\left|d_{1}\right|\left|\eta_{1}^{\dagger}\right|+\left|\frac{d_{2}}{d_{1}}\right| \Delta_{e}\right)
\end{aligned}
$$

so that the following proposition will be valid.

Proposition 3.3 If $\eta_{2}=\eta_{2}^{*}$ is given as in (23), then the tracking error of the system (6) will converge to the dead-zone range $\left[-\Delta_{e}, \Delta_{\epsilon}\right]$ globally and exponentially.

However, the controller given in (23) again faces the problem with discontiriuity so that, similar to (21), we replace the controller (23) with a smooth compensator with the dead-zone range $\left[-\Delta_{\eta_{1}}, \Delta_{\eta_{1}}\right]$ as follows:

$$
\eta_{2}^{\dagger}=\lambda_{1} \eta_{1}^{\dagger}+k_{2}\left(e_{o}, \tilde{\eta_{1}}, \alpha, \Delta_{\varepsilon}, \Delta_{\eta_{1}}\right)
$$

where

$$
k_{2}= \begin{cases}k_{21}(t) \widetilde{\eta_{1}}+k_{22}(t) \operatorname{sgn}\left(\widetilde{\eta_{1}}\right), & \widetilde{\eta_{1}} \notin\left[-\Delta_{\eta_{1}}, \Delta_{\eta_{1}}\right] \\ k_{s_{2}}\left(e_{o}, \widetilde{\eta_{1}}, \alpha, \Delta_{e}, \Delta_{\eta_{1}}\right), & \text { otherwise; }\end{cases}
$$

with $k_{s 2}(\cdot)$ being a smooth function in order to make $\eta_{2}^{\dagger}$ smooth. By the same token, the controller design can back steps to the equation containing the real control input:

$$
\dot{\eta}_{\rho-1}=-\lambda_{\rho-1} \eta_{\rho-1}+\delta(y) u
$$

However, unfortunately, the above controller, can realize its designated controller only up to the corresponding dead-zone ranges. For example, the controller $\eta_{2}^{\dagger}$ given in (25) can realize $\eta_{1}^{\dagger}$ given in (21) only up to the dead-zone range $\left[-\Delta_{\eta_{1}}, \Delta_{\eta_{1}}\right]$. This fact results in that the former proposition will no longer hold. Thus, we will require additional compensators to compensate for the backward dead-zone ranges, yielding the following set of designated controllers (desired filter states):

$$
\begin{aligned}
\eta_{1}^{\dagger} & =\operatorname{sgn}\left(d_{1}\right)\left(k_{1}+k_{\delta 1} e_{o}\right) \\
\eta_{2}^{\dagger} & =\lambda_{1} \eta_{1}^{\dagger}+k_{2}+k_{\delta 2} \tilde{\eta_{1}} \\
& \vdots \\
\eta_{\rho-1}^{\dagger} & =\lambda_{c-2} \eta_{\rho-2}^{\dagger}+k_{\rho-1}+k_{\delta \rho-1} \\
\delta(y) u & =\eta_{\rho}^{\dagger}=\lambda_{\rho-1} \eta_{\rho-1}^{\dagger}+k_{\rho},
\end{aligned}
$$

where $k_{3}(\cdot), \cdots, k_{\rho}(\cdot)$ and $k_{\delta 1}(\cdot), \cdots, k_{\delta \rho-1}(\cdot)$ are the designated compensators to be defined later, the tracking errors are defined as $\widetilde{\eta_{2}}=\eta_{2}^{\dagger}-\eta_{2}, \cdots, \widetilde{\eta_{\rho-1}}=\eta_{\rho-1}^{\dagger}-\eta_{\rho-1}$ corresponding to the dead-zone ranges $\left[-\Delta_{\eta_{2}}, \Delta_{\eta_{2}}\right], \cdots,\left[-\Delta_{\eta_{\rho-1}}, \Delta_{\eta_{\rho-1}}\right]$, respectively, and

$$
\widetilde{\eta_{j}}= \begin{cases}\widetilde{\eta_{j}} & \text { as } \tilde{\eta_{j}}<-\Delta_{\eta_{j}} \text { or } \widetilde{\eta_{j}}>\Delta_{\eta_{j}} \\ 0, & \text { otherwise (i.e. } \left.\tilde{\eta_{j}} \in\left[-\Delta_{\eta_{j}}, \Delta_{\eta_{j}}\right]\right)\end{cases}
$$

so that

$$
\tilde{\eta}_{j}=\dot{\eta_{j}} \text { for }{\widetilde{\eta_{j}}}_{\Delta} \neq 0
$$

for $j=1, \cdots, \rho-1$

Then, the following theorem is valid. 
Theorem 3.1 If the control law $\delta(y) u=\eta_{\rho}^{\dagger}$ is given as in (28), then the system state in (4) is guaranteed to be bounded and the tracking error of the system (6) will converge to the dead-zone range $\left[-\Delta_{e}, \Delta_{e}\right]$ globally and exponentially.

Remark: While the given designated controllers are not smooth, then the subsequent designated controllers will be hardened with high-gain since the controllers contain the differential terms, e.g., $\frac{\partial \eta_{1}^{\dagger}}{\partial e_{0}}, \frac{\partial \eta_{2}^{\dagger}}{\partial e_{0}}, \frac{\partial \eta_{2}^{\dagger}}{\partial \eta_{1}}, \cdots, \frac{\partial \eta_{\rho-1}^{\dagger}}{\partial \eta_{\rho-2}}[3]$. Besides, it is difficult to realize these designated controllers when considering the uncertainties of the controlled system. In the following section, we will propose an adaptive fuzzy variable structure control to solve the above mentioned problem.

\section{Adaptive Fuzzy Variable Structure Control}

Consider

fuzzy controller input (vector) $u_{f}=\left[u_{f_{1}}, \cdots, u_{f_{\rho}}\right]^{T}$, consisting of $\rho$ multi-input single-output (MISO) fuzzy controls, which are respectively characterized by

$$
\begin{aligned}
u_{f_{1}} & \triangleq u_{f_{1}}\left(w_{1}\right): \Omega_{w_{1}} \rightarrow \Re \\
u_{f_{2}} & \triangleq u_{f_{2}}\left(w_{1}, w_{2}\right): \Omega_{w_{1}} \times \Omega_{w_{2}} \rightarrow \Re \\
& \vdots \\
u_{f_{i}} & \triangleq u_{f_{i}}\left(w_{1}, \cdots, w_{i}\right): \Omega_{w_{1}} \times \Omega_{w_{2}} \times \cdots \times \Omega_{w_{i}} \rightarrow \Re \\
& \vdots \\
u_{f_{\rho}} & \triangleq u_{f_{\rho}}\left(w_{1}, \cdots, w_{\rho}\right): \Omega_{w_{1}} \times \cdots \times \Omega_{w_{\rho}} \rightarrow \Re
\end{aligned}
$$

where $u_{f_{i}}\left(w_{1}, \cdots, w_{i}\right)$ is the $i$-th fuzyy controller, $w_{1}, \cdots, w_{\rho}$ are defined as input fuzzy variables

$$
w=\left[w_{1}, \cdots, w_{\rho}\right]^{T}=\left[e_{\boldsymbol{o}}, \tilde{\eta}_{1}, \cdots, \tilde{\eta}_{\rho-1}\right]^{T}
$$

and $\Omega_{w_{1}} \equiv\left[-\Upsilon \Delta_{1}, \Upsilon \Delta_{1}\right], \Omega_{w_{2}} \equiv\left[-\Upsilon \Delta_{2}, \Upsilon \Delta_{2}\right], \cdots, \Omega_{w_{\rho}} \equiv$ [- $\Upsilon \Delta_{\rho}, \Upsilon \Delta_{\rho}$ ], with $\Upsilon$ being an arbitrarily large positive integer, and $\Delta_{1}, \cdots, \Delta_{\rho}$ being some positive real numbers. Here, each of the membership functions is given as an $m$-th $(m \geq 2)$ order multiple dimension central B-spline function (as depicted in Fig.1), of which the $j$-th dimension is defined as follows:

$N_{m j}(x)=\sum_{k=0}^{m+1} \frac{(-1)^{k}}{m !}\left(\begin{array}{c}m+1 \\ k\end{array}\right)\left[\left(x+\left(\frac{m+1}{2}-k\right) \Delta_{j}\right)_{+}\right]^{m}$

where we use the notation

$$
x_{+}:=\max (0, x)
$$

The $m$-th order B-spline type of membership function has the following properties:

- an $(m-1)$-th order continuously differentiable function, i.e., $N_{m j}(x) \in C^{m-1}$

- local compact support, i.e., $N_{m_{j}}(x) \neq 0$ only for $x \in$ $\left[-\frac{m+1}{2} \Delta_{j}, \frac{m+1}{2} \Delta_{j}\right]$

- $N_{m j}(x)>0$ for $x \in\left(-\frac{m+1}{2} \Delta_{j}, \frac{m+1}{2} \Delta_{j}\right)$

- symmetric with respect to the center point (zero point)

- $\sum_{i_{1}=-\infty}^{\infty} \cdots \sum_{i_{j}=-\infty}^{\infty} N_{m_{1}}\left(x-i_{1} \Delta_{1}\right) \cdots N_{m_{j}}\left(x-i_{j} \Delta_{j}\right)=$ $1, \forall x \in \Re, j \in \mathcal{Z}^{+}$

Then the membership functions for the $j$-th fuzzy variable $w_{j}$ are defined as follows:

$$
\mu_{j_{i}}\left(w_{j}\right)=N_{m_{j}}\left(w_{j}-i \Delta_{j}\right), i=-\Upsilon, \cdots, 0, \cdots, \Upsilon
$$

whose compact support is given as:

$$
\Omega_{w_{j i}}=\left[\left(i-\frac{m+1}{2}\right) \Delta_{j},\left(i+\frac{m+1}{2}\right) \Delta_{j}\right], i=-\Upsilon, \cdots, \Upsilon
$$

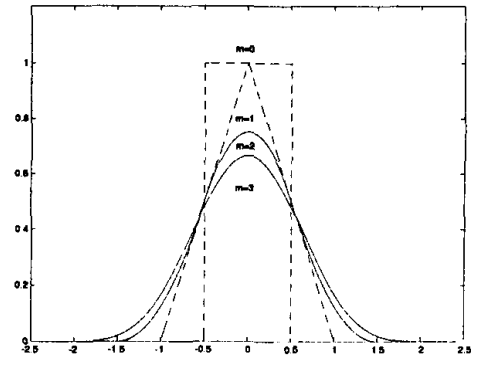

Figure 1: The $m$-th order B-spline basis for $m=0,1,2$, and 3

for $j=1, \cdots, \rho$ which means that $w_{j} \in \operatorname{int}\left(\Omega_{w_{j}}\right)$ implies that $\mu_{j_{i}}\left(w_{j}\right)>0$. Apparently, we can get $\Omega_{w_{j}} \equiv U_{i \in\{-\Upsilon, \cdots, \Upsilon\}} \Omega_{w_{j_{i}}} \equiv$ $\left[-\Upsilon \Delta_{j}, \Upsilon \Delta_{j}\right]$. Besides, it is possible that $\Omega_{w_{j}} \cap \Omega_{w_{j k}} \neq \emptyset$, for some $i \neq k$, i.e., $w_{j}$ can simultaneously fall into several compact supports. It is interesting to note that the indices labeling those supports can be reexpressed as:

$$
\begin{aligned}
I_{c j}\left(w_{j}\right) & \equiv\left\{i: w_{j} \in \operatorname{int}\left(\Omega_{w_{j i}}\right), i \in \mathbb{Z}, \Upsilon \leq i \leq \Upsilon\right\} \\
& \equiv\left\{i: \Omega_{w_{j_{i}}} \subset \Omega_{c_{j}}\left(w_{j}\right)\right\}
\end{aligned}
$$

where $\Omega_{c_{j}}\left(w_{j}\right)$ is the union set of those compact supports, defined as follows:

$$
\Omega_{c_{j}}\left(w_{j}\right) \equiv \cup_{i \in I_{c_{j}}\left(w_{j}\right)} \Omega_{w_{j i}}
$$

which means that $i \in I_{c j}\left(w_{j}\right)$ if and only if $w_{j} \in \Omega_{c_{j}}\left(w_{j}\right)$.

As a general representation of the MISO fuzzy controller with center average defuzzifier, inference with product compositional operator, and singleton fuzzifier [24], we can represent the above fuzzy controllers as follows:

$$
\begin{aligned}
& u_{f_{1}}=\frac{\sum_{i_{1}=-\Upsilon}^{\Upsilon} \mu_{1_{i_{1}}}\left(w_{1}\right) \theta_{i_{1}}}{\sum_{i_{1}=-\Upsilon}^{\Upsilon} \mu_{1_{1}}\left(w_{1}\right)}=\sum_{i_{1}=-\Upsilon}^{\Upsilon} \nu_{i_{1}}\left(w_{1}\right) \theta_{i_{1}}=\theta^{(1)^{T}} \nu^{(1)} \\
& u_{f_{k}}=\frac{\sum_{i_{1}=-\Upsilon}^{\Upsilon} \cdots \sum_{i_{k}=-\Upsilon}^{\Upsilon} \mu_{i_{i_{1}}}\left(w_{1}\right) \cdots \mu_{k_{i_{k}}}\left(w_{k}\right) \theta_{i_{1} i_{2} \cdots i_{k}}}{\sum_{i_{1}=-\Upsilon}^{\Upsilon} \cdots \sum_{i_{k}=-\Upsilon}^{\Upsilon} \mu_{1_{i_{1}}}\left(w_{1}\right) \cdots \mu_{k_{k}}\left(w_{k}\right)} \\
& =\sum_{i_{1}=-\Upsilon}^{\Upsilon} \cdots \sum_{i_{k}=-\Upsilon}^{\Upsilon} \nu_{i_{1} \cdots i_{k}}\left(w_{1}, \cdots, w_{k}\right) \theta_{i_{1} \cdots i_{k}} \\
& =\theta^{(k)^{T}} \nu^{(k)} \\
& u_{f \rho}=\frac{\sum_{i_{1}=-\Upsilon}^{\Upsilon} \cdots \sum_{i_{\rho}=-\Upsilon}^{\Upsilon} \mu_{1_{i_{1}}}\left(w_{1}\right) \cdots \mu_{\rho_{i_{\rho}}}\left(w_{\rho}\right) \theta_{i_{1} i_{2} \cdots i_{\rho}}}{\sum_{i_{1}=-\Upsilon}^{\Upsilon} \cdots \sum_{i_{\rho}=-\Upsilon}^{\Upsilon} \mu_{1_{i_{1}}}\left(w_{1}\right) \cdots \mu_{\rho_{i_{\rho}}}\left(w_{\rho}\right)} \\
& =\sum_{i_{1}=-\Upsilon}^{\Upsilon} \cdots \sum_{i_{\rho}=-\Upsilon}^{\Upsilon} \nu_{i_{1} \cdots i_{\rho}}\left(w_{1}, \cdots, w_{\rho}\right) \theta_{i_{1} \cdots i_{\rho}} \\
& =\theta^{(\rho)^{T}} \nu^{(\rho)}
\end{aligned}
$$

where $i_{1}, \cdots, i_{k}, \cdots, i_{\rho}$ are integer indices, $\nu_{i_{1} \cdots i_{k}}\left(w_{1}, \cdots, w_{k}\right)$ is the fuzzy basis function of the $k$-th fuzzy controller associated with the indices $i_{1} \cdots i_{k}$, defined as follows:

$$
\begin{aligned}
& \nu_{i_{1} \cdots i_{k}}=\frac{\mu_{1_{1}}\left(w_{1}\right) \cdots \mu_{k i_{k}}\left(w_{k}\right)}{\sum_{i_{1}=-\Upsilon}^{\Upsilon} \cdots \sum_{i_{k}=-\Upsilon}^{\Upsilon} \mu_{1_{i_{1}}}\left(w_{1}\right) \cdots \mu_{k i_{k}}\left(w_{k}\right)} \\
& =\frac{\mu_{1_{i_{1}}\left(w_{1}\right) \cdots \mu_{k i_{k}}\left(w_{k}\right)}}{\sum_{i_{1} \in I_{c 1}\left(w_{1}\right)} \cdots \sum_{i_{k} \in I_{c k}\left(w_{k}\right)} \mu_{1_{i_{1}}}\left(w_{1}\right) \cdots \mu_{k_{i_{k}}}\left(w_{k}\right)} \text {, }
\end{aligned}
$$




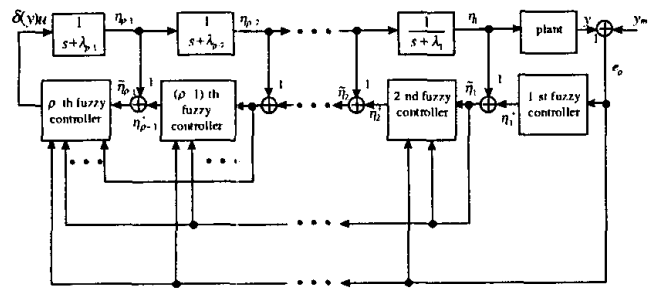

Figure 2: The block diagram of the closed-loop system with $\rho \geq 2$

$\theta_{i_{1} \cdots i_{k}}$ is the parameter of the $k$-th fuzzy controller associated with the indices $i_{1} \cdots i_{k}, \nu^{(k)}$ and $\theta^{(k)}$ are the vectors consisting of $\nu_{i_{1} \cdots i_{k}}$ and $\theta_{i_{1} \cdots i_{k}}$ for $i_{1}=-\Upsilon, \cdots, \Upsilon, \cdots, i_{k}=-\Upsilon, \cdots, \Upsilon$, respectively. The block diagram of the overall closed-loop system is depicted in Fig. 2. Furthermore, we realize the adaptive fuzzy variable structure control law as an integrated control consisting of the former fuzzy control vector $u_{f}$ and a supervised control vector $\bar{\eta}=\left[\overline{\eta_{1}}, \cdots, \overline{\eta_{\rho}}\right]^{T}$ as follows:

$$
\begin{array}{r}
\text { If } w_{1} \in \Omega_{w_{1}} \text {, then } \eta_{1}^{\dagger}=\operatorname{sgn}\left(d_{1}\right)\left(u_{f_{1}}+k_{f_{1}} w_{1}\right) ; \text { otherwise } \eta_{1}^{\dagger}=\overline{\eta_{1}} \\
\text { If } w_{2} \in \Omega_{w_{2}} \text {, then } \eta_{2}^{\dagger}=\lambda_{1} \eta_{1}^{\dagger}+u_{f_{2}}+k_{f_{2}} w_{2} \text {; otherwise } \eta_{2}^{\dagger}=\overline{\eta_{2}}
\end{array}
$$

If $w_{\rho} \in \Omega_{w_{\rho}}$, then $\eta_{\rho}^{\dagger}=\lambda_{\rho-1} \eta_{\rho-1}^{\dagger}+u_{f_{\rho}}+k_{f_{\rho}} w_{\rho} ;$ otherwise $\eta_{\rho}^{\dagger}=\overline{\eta_{\rho}}$ where $k_{f_{1}}, \cdots, k_{f_{\rho}}$ are some positive constants and

$$
\begin{aligned}
\overline{\eta_{1}} & =\operatorname{sgn}\left(d_{1}\right) \overline{k_{1}}(t) \\
\overline{\eta_{2}} & =\lambda_{1} \eta_{1}^{\dagger}+\overline{k_{2}}(t) \\
\vdots & \\
\overline{\eta_{\rho}} & =\lambda_{\rho-1} \eta_{\rho-1}^{\dagger}+\overline{k_{\rho}}(t)
\end{aligned}
$$

Here, the dead-zone ranges are defined as the compact supports of membership functions $\mu_{1} \Upsilon\left(u_{1}\right), \cdots, \mu_{\rho}\left(w_{\rho}\right)$, namely,

$$
\begin{array}{r}
\Omega_{w_{10}} \equiv\left[-\frac{m+1}{2} \Delta_{1}, \frac{m+1}{2} \Delta_{1}\right] \equiv\left\{-\Delta_{w_{1}}, \Delta_{w_{1}}\right] \equiv\left\{-\Delta_{e}, \Delta_{e}\right] \\
\Omega_{w_{\rho_{0}}} \equiv\left[-\frac{m+1}{2} \Delta_{\rho}, \frac{m+1}{2} \Delta_{\rho}\right\} \equiv\left[-\Delta_{w_{p}}, \Delta_{w_{p}}\right] \equiv\left[-\Delta_{\eta_{\rho-1}}, \Delta_{\eta_{\rho-1}}\right]
\end{array}
$$

Then, the following proposition can be established.

Proposition 4.1 If the control law $\delta(y) u=\eta_{\rho}^{\dagger}$ is given as in the equations (37), (38) and (39), then there exist a class of the fuzzy controller vector $u_{f}$ given as in (37) which can drive the tracking error of the system $(6), w_{1}\left(=e_{o}\right)$, into the dead-zone range $\left[-\Delta_{w_{1}}, \Delta_{w_{1}}\right]$ globally and exponentialiy.

Now, define the optimal parameter vector of the $j$-th fuzzy controller as follows:

$$
\begin{aligned}
& \theta^{(j) *}=\operatorname{argmin}\left\{\sup _{w_{1} \in \Omega_{w_{1}}, \cdots, w_{i_{j}-1} \in \Omega_{w_{i_{j}-1}}, w_{j} \in \Omega_{w_{i_{j}}} \backslash \Omega_{w_{j}}}\right. \\
& \sum_{i_{1}=-\Upsilon}^{\Upsilon} \ldots \sum_{i_{j}=-\Upsilon}^{\Upsilon} \sum_{i_{j}=-\Upsilon}^{\Upsilon} \nu_{i_{1} \cdots i_{j}} \theta_{i_{1} \cdots i_{j}} \operatorname{sgn}\left(w_{j}\right) \\
& \geq\left\{k_{j}(t)+k_{\delta j}(t) w_{j} \mid\right\}
\end{aligned}
$$

It is, however, that $\theta^{(j) *}$ may not easily be available due to the complexity of $k_{j}(t)$ and $k_{\delta j}(t), j=1, \cdots, \rho$. Therefore, the following adaptive law to update the parameters vector $\theta^{(j)}$ will be
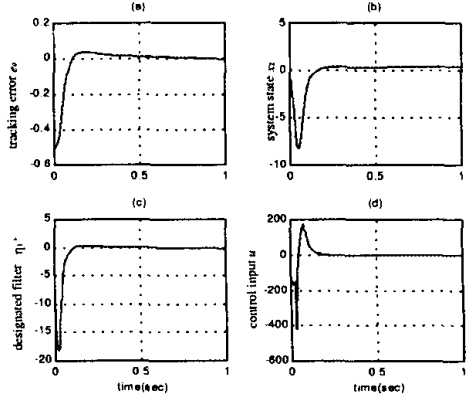

Figure 3: The simulation results for $\rho=2$

necessary so that the tracking error can be driven toward the deadzone range:

$$
\begin{aligned}
& \dot{\theta}^{(1)}= \begin{cases}r d_{1} \nu(1)\left(w_{1}\right) w_{1} \Delta, & \text { for } w_{1} \in \Omega_{w_{1}} \\
0, & \text { otherwise }\end{cases} \\
& \dot{\theta}^{(2)}= \begin{cases}r_{\nu}(2)\left(w_{1}, w_{2}\right) w_{2} \Delta, & \text { for } w_{1} \in \Omega_{w_{1}}, w_{2} \in \Omega_{w_{2}} \\
0, & \text { otherwise }\end{cases} \\
& \dot{\theta}^{(\rho)}= \begin{cases}r_{\nu}(\rho)\left(w_{1}, \ldots, w_{\rho}\right) w_{\rho}, & \text { for } w_{1} \in \Omega_{w_{1}}, \cdots, w_{\rho} \in \Omega_{w_{\rho}}(42) \\
0, & \text { otherwise }\end{cases}
\end{aligned}
$$

where $w_{1 \Delta}=e_{o \Delta}, w_{2 \Delta}=\eta_{1 \Delta}, \cdots, w_{\rho \Delta}=\eta_{\rho-1}$. Based on the control law in (37), (38), (39), and the adaptive law (42), the system can be shown to achieve appropriate output error convergence into a prespecified dead-zone range. This is summarized and proved in the following theorem.

Theorem 4.1 If adaptive fuzzy variable structure control law is given as in the equations (37), (38), (39) with the adaptive law (42), then the output tracking error of the system (6) will be driven to the dead zone range $\left[-\Delta_{w_{1}}, \Delta_{w_{1}}\right]$ globally and asymptotically.

Remark: The fuzzy controller (37) with the adaptive law ( 42 ) possesses the following advantages:

- locally weighted fuzzy controller: Only rules supported by compact set $\Omega_{c j}$ are required to be updated, and hence, those rules are locally weighted.

- smooth fuzzy controller: Apparently, the fuzzy controller (37) can behave as a smoother controller provided the differential terms $\frac{\partial u_{f}}{\partial w_{1}}, \frac{\partial u_{f_{2}}}{\partial w_{1}}, \cdots, \frac{\partial u_{f_{\rho}}}{\partial w_{\rho}}$ can be made small, which then requires that smoother membership functions are adopted. Thus, hardening the controllers with high-gain in the backstepping procedure can be naturally avoided here, if we can choose the membership functions to be even smoother high order B-spline functions.

Consider the system (see [2]) as follows:

$$
\begin{aligned}
\dot{x}_{1} & =x_{2}+x_{1}^{\alpha} \\
\dot{x}_{2} & =u \\
y & =x_{1}
\end{aligned}
$$

In this example, $\alpha=2$ is assumed to be unknown. The system is a relative degree $\rho=2$ system and a stable filter (7) is given as $\frac{1}{s+1}$. The desired trajectory is given as $y_{m}(t)=\frac{1}{s^{2}+2 s+1} r_{m}(t)$, and $r_{m}(t)$ is given as a step input, i.e., $r_{m}(t)=1$. The developed adaptive fuzzy variable structure control to be applied to the system described in the following 4.1. The fuzzy controller 


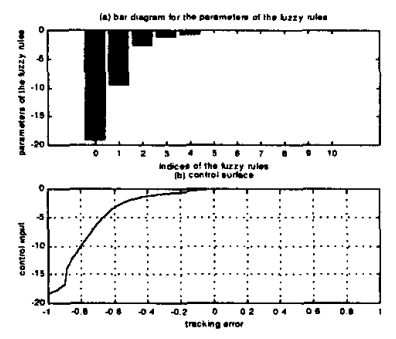

Figure 4: The bar diagram for the parameters of the fir: fuzzy controller and control surface

is synthesized as follows: the first fuzzy controller for the desi nated filter state $\eta_{1}$ takes the tracking error $e_{o}$ as its single inp variable $w_{1}$ whereas the second fuzzy controller takes the tracki error $e_{o}$ and output of the first fuzzy controller as two input val ables $\left(w_{1}, w_{2}\right)$. The fuzzy rule number of the first fuzzy controll is equal to $2 \Upsilon+1=11$ and that of the second fuzzy controller equal to $11 \times 11=121$. Moreover, $k_{f_{1}}=k_{f_{2}}=10$ is assigned. $\mathrm{Fi}$ 3 shows that the tracking errors can converge into the dead-zol range. Figure 4(a) shows a bar diagram for the final paramete of the first fuzzy controller after updating. Apparently, only a he of parameters of fuzzy rules had been updated since $e_{o}$ almo stays within the region $e_{o}<0$ durring the task running. Fig. 4 (') shows the smooth control surface of the first fuzzy controller. Fi ure 5(a) shows a 3-dimension bar diagram of the final paramete of the second fuzzy controller after updating and Fig. 5(b) shor a 3-dimension plot for smooth control surface of the first fuz: controller.

\section{Conclusion}

In this paper, we proposed a novel adaptive fuzzy variat structure control via backstepping for a class of SISO nonline systems which can solve the traditional model reference adapti control problem in the presence of system uncertainties. It was ri orously proved that the stability of the overall system is assur and the tracking error can be driven to the designated dead-zol. range. Besides, with undesirable chattering from the " hard" high-gain control lawscan be avoided due to the adoption of the smooth B-spline-type membership functions.Satient features of the present work includes that the involved rules are locally weighted and the output control is rather smooth.

\section{References}

[1] Riccardo Marino and Patrizio Tomei " Global Adaptive Output-Feedback Control of Nonlineaz Sybtem. Part 1: Linear Parameterizatic Jamtary 199.8

[2] Riccardo Marino and Patrizio Tomei “ Global Adaptive Output-Feedback Control of Nonlinear System, Part II: Nonlinear Parameterization," IEE

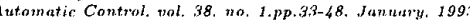

[3] R. A. Freeman and P. V. Kokotovic, “Design of 'Softer' Robust Nonlinear

[4] R. A. Freeman and P. V. Kokotovic. "Tracking Controllers for Systems Linear in the Unmodeled States." Altitinntica. vol. 32. no. 5. pp. 735-745. 1996

[5] S. Shankar Sastry and Alberto Isidori, "Adaptive Control of Linearizable Systems," IEEE Trangations on Automatic Control. wol. 34. no. 11.pp. 1123-1131.

[6] David G. Taylor. P. V. Kokotovic and R. Marino," Adaptive Regulation of Nonlisear Systems with Unmodeled Dynamics," IEEE Trunasuctions on Antomatic Control. 1ol. 34. 7ro. 4.pp. 405-412. 1989

(7) Ioannis Kanell., P. V. Kokotovic and A. S. Morse," Systematic Design of Adaptive Controllers for Feedback Linearizable Systems," IEEE Transantion. on Automutic Control. wol. 36. no. 11,pp. 1241-125.1991

[8] Miroslav Krstic. and P. V. Kokotovic, "Adaptive Nonlinear Output-Feedback Schemes with Marino-Tomei Controller," IEEE Trnnsactions on Automatic Contrel. vol. 41. nn. 2.pp. 274-280., 1996

[9] C. J. Chien and L. C. Fu, "An Adaptive Variable Structure Control for a Class of Nonlinear System," Syst. Contr. istt.. Vol. 21. no. 1. pp. 49.5\%, 199.3

[10] R. A. Decarlo.. S. H. Zak, and G. P. Matthews. "Variable Structure Control of Nonlinear Multivariable Systems : a tutorial "Proceetings of the IEEE. Wol.
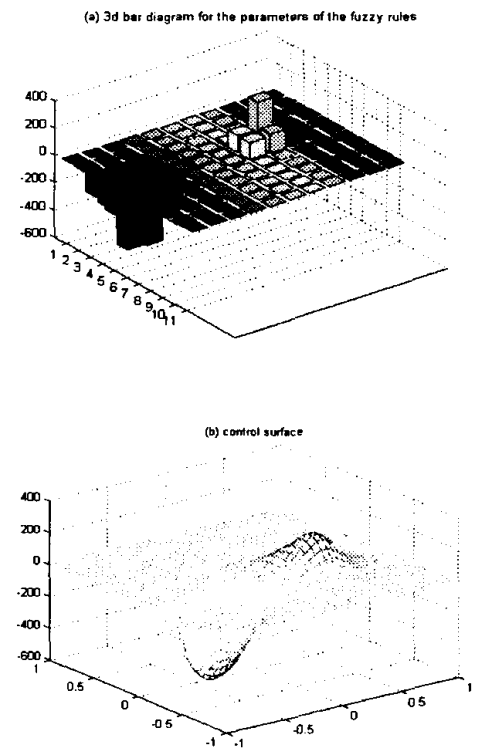

Figure 5: The 3d bar diagram for the parameters of the second fuzzy controller and control surface

[11] D. S. Yoo and M. J. Chung. "A Variable Structure Control with Simple Adaptation Laws for Upper Bounds on

[12] z. Qu, "Robust Control of Nonlinear Uncertainties Systems under GeneralZ. Qu, "Robust Control of Nonlinesr Uneertainties Systems
ized Matching Conditions," Automatica. "ol. 29. pp. 985-998. 199.3

[13] C. M. Kwan., " Sliding Mode Control of Linear Systems with Mismatched

[14] G. Song, Y. Wang, L. Cai, and R. W. Longman "A Sliding-Mode Brsed Smooth Adaptive Robust Controller for Friction Compensation," Procending of

[15] V.S. C. Raviraj and P. C. Sen, "Comparative Study of Proportional-1ntegral "Sliding Mode, and Fuzzy Logic Controllers for Power Converters," IEEE Trun

[16] Jacob $S$, Glower and Jeffery Muunighan, "Designing Fuzzy Controllers from a variable structures standpoint," IEEE Trans. on Fuzzy Systems. vol.5. no. 1. pp.1.38-

[17] S.-C. Lin and Y.-Y. Chen, "Design of Self-Learning Fuzzy Sliding Mode Controllers Based on Genetic Algorithms," Fuzzy Sets and Syatems nal.86. no.2. pp. 139.153. Mar. 1998

[18] J. C. Wu and T. S. Liu, "Fuzzy Control Stabilization with Applications to Motorcycle Control," IEEE Thrms. on Systems. Man. ant Cybernetics. PartB: Cybernetirs. nol. 26. no. 6. pp.836-847, Der, 1996

[19] Feng-Yih Hsu and Li-Chen Fu, "Adaptive Robust Fuzzy Control for Robot Adobotics and Automation. pp. 629-634. 1994

[20] Feng-Yih Hsu and Li-Chen Fu, "A New Design of Adaptive Fuzzy Hybrid Force/Position Controller for Ro
und Automation. pp. 86.3-868.1995

[21] Feng-Yih Hsu and Li-Chen Fu. "An Adaptive Fuzzy Hybrid Control for Robot Manipulators Following Contours of an Uncertain Object". JEEE Conferener on Manipulators Following Contours of an U1
Rabatics and Autamation, pp. 22.32-22.37. 1996

[22] Feng-Yih Hsu and Li-Chen Fu. "Intelligent Robot Deburring Using Adaptive Fuzzy Hybrid Control"

[23] R. M. Sanner and J-J E. Slotine, "Gaussian Networks for Direcet Adnptive R. M. Sanner and J-J E. Slotine, "Gaussian Networks for Direcet
Control", IEEE. Trans. on. Neural Networks, vol. 3, no, 6. pp. 8.37-863. 1992.

[24] L.-X. Wang, Adaptive Fuzzy Systems and Control: Design and Stability anal-

[25] F.-Y. Hsu and L.-C. Fu, "Recent Progress in Fuzzy Control," A Chapter in Control Problems in Robotice and Automatic

[26] K. Chui Charles, An Introduction to Wauretes, Wavelet Analysis and Its Application, vol. 1, pp. 80-90, 1991 [27] Jonas SJO., Qin. Zhang., Len. Ljung. .... "Nonlinear Black-box Modeling
in System Identification: a Unified Overview," Antomatica. vol. 31. no. 12. pp.
1725.1750 .1995

[28] Anatoli Judi., Hakan Hjal., Albert Benv. ..., "Nonlinear Black-box Models in System Identification: Mathematical Foundations," Automatica. vol. 31. no.
12. pp. 1725-1750.1995 